\title{
Combustion Response of Ammonium Perchlorate
}

\author{
Michael Shusser* and Fred E. C. Culick ${ }^{\dagger}$ \\ California Institute of Technology, Pasadena, California 91125 \\ and \\ Norman S. Cohen \\ Cohen Professional Services, Redlands, California 92373
}

\begin{abstract}
A modified Price-Boggs-Derr model is applied to compute the linear and nonlinear combustion response properties of monopropellant ammonium perchlorate (AP). The kinetics constants were changed to achieve good agreement with response function data as well as with steady-state data. The numerical method was first validated with the classical theory. Computations using the Levine and Culick boundary condition in the limit of small perturbations were compared with the exact mathematical solution for linear response, and the effect of perturbation amplitude was explored. Then, using the AP model for the boundary condition, various linear and nonlinear computations were performed. Supplemental mathematical analyses relate the AP model to the basic two parameters of the classical theory and show the key factors determining the nature of the combustion response.
\end{abstract}

\section{Nomenclature}

$=$ dimensionless parameter characteristic of surface decomposition

$=$ kinetics prefactor for the ammonium perchlorate (AP) flame

$=$ kinetics prefactor for AP surface decomposition

$=$ kinetics prefactor for exothermic condensed phase reaction

$=$ dimensionless parameter characteristic of the coupling of the gas phase and the surface

$=$ specific heat of the gas

$=$ specific heat of the condensed phase

$=$ activation energy of the AP flame

$=$ activation energy of AP surface decomposition

$=$ activation energy of exothermic condensed phase reaction

$=$ frequency of oscillations

$=$ dimensionless net exothermicity of the condensed phase

$=$ dimensionless heat release from the AP flame

$=$ thermal conductivity of the gas

$=\mathrm{AP}$ mass flux

$=$ burn rate pressure exponent

$=$ oxidizer

$=$ dimensionless pressure, $p / \bar{p}$

$=$ pressure

$=$ heat content of the adiabatic AP flame

$=$ heat of exothermic condensed phase reaction

$=$ heat release from the AP flame

$=$ dimensionless $\mathrm{AP}$ burn rate, $r_{\mathrm{ox}} / \bar{r}_{\mathrm{ox}}$

$=$ pressure-coupledresponse function

$=$ universal gas constant

$=\mathrm{AP}$ burn rate

$=\mathrm{AP}$ flame temperature

$=\mathrm{AP}$ surface temperature
Presented as Paper 2000-3694 at the AIAA 36th Joint Propulsion Conference, Huntsville, AL, 16-19 July 2000; received 9 August 2000; revision received 14 May 2001; accepted for publication 6 June 2001. Copyright (C) 2001 by the authors. Published by the American Institute of Aeronautics and Astronautics, Inc., with permission. Copies of this paper may be made for personal or internal use, on condition that the copier pay the $\$ 10.00$ per-copy fee to the Copyright Clearance Center, Inc., 222 Rosewood Drive, Danvers, MA 01923; include the code 0001-1452/02 \$10.00 in correspondence with the CCC.

*Postdoctoral Scholar, Mechanical Engineering. Member AIAA.

${ }^{\dagger}$ Professor, Mechanical Engineering and Jet Propulsion. Fellow AIAA.

* Subcontractor. Associate Fellow AIAA.

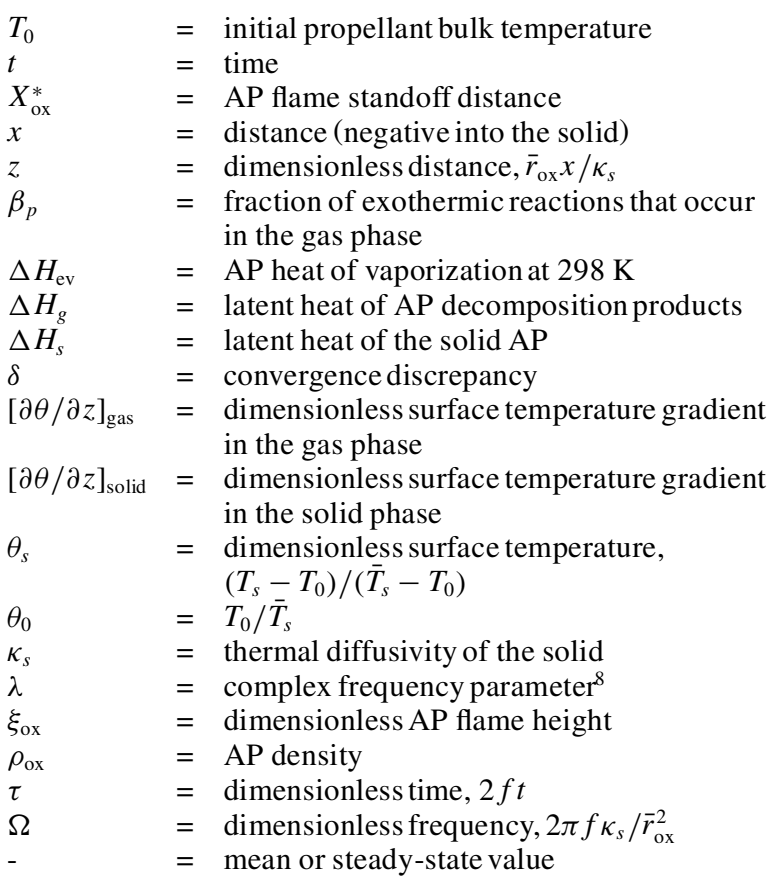

\section{Introduction}

$\mathbf{T}$ HE objective of the Multidisciplinary University Research Initiative (MURI) on combustion instability is to develop understanding and capabilities that will assure the future stability of solid rocket motors employing advanced energetic propellants. A starting point is to work with ammonium perchlorate (AP) composite propellants because of their long history and continuing interests for the foreseeable future. Furthermore, the axial-mode instabilities of AP composite propellants present the most challenging solid rocket motor instability problem. The most frequent encounters with axialmode instabilities are with AP propellants, and the convenience of particle damping by additivesto suppresshigh-frequencytangentialmode instabilities is not available at the lower axial-mode frequencies. Moreover, the axial modes are those that are associated with the important gasdynamic mechanism of vortex shedding.

The general approach is to achieve a numerical simulation of the internal flowfields of solid motors, which necessarily includes the coupling of the motor chamber gasdynamics with the combustion process at the boundaries of the flowfield. The standard work in this area has been the Levine and Baum nonlinear instability code, ${ }^{1}$ which coupled one-dimensional gasdynamics with a simple and heuristic representation of the combustion. Although remarkable in 
its ability to describe features of nonlinear instability observed in research motors, the Levine and Baum one-dimensional model is inadequate to represent most motor geometries of practical interest or to describe vortex shedding, and its combustion model does not contain mechanisms to isolate the key propellant variable of AP particle size or the key flow variable of turbulenceinteraction. Thus, there is need to evolve to two-dimensional gasdynamics coupled with a comprehensive composite propellant model.

The combustion model used in this work is the Cohen and Strand ${ }^{2}$ model developed in the 1980s. It has already been used to explain effects of AP particle size, pressure, and crossflow on combustion response properties, in a general qualitative way, through the application of Zeldovich-Novozhilov methodology (see Refs. 3 and 4). However, this model has not yet been incorporatedinto a numerical scheme that would take full advantage of its mechanistic features, such as the coupled scheme envisioned in the MURI objective. In doing so, it is necessary to incorporate a mechanistic model of turbulence interaction. Beddini's model ${ }^{5}$ is being used for this purpose.

As with composite propellant combustion modeling work in the past, new developments begin with a study of monopropellant AP. The paper is limited to pressure coupling because there are no erosive burning or acoustic erosivity data for AP. It is only recently that combustion response function data have been acquired for AP (Ref. 6), which are important to evaluate the validity of the mechanistic features of the model as a prerequisite for the composite propellant modeling. Pressure oscillations are imposed as external inputs to the nonsteady combustion model computer code.

\section{AP Model}

The model for AP is the Price-Boggs-Derr model ${ }^{7}$ as adapted for use in a composite propellant model. ${ }^{2}$ It successfully predicted the burn rates, pressure exponents, and temperature sensitivities of AP over broad ranges of pressure and conditioning temperature. However, its initial use in the numerical scheme to compute response functions was not successful. For reasons to be explained later, it predicteda response function curve that was flat and with low values. In relation to the classical two-parameter $(A$ and $B)$ theory of linear combustion response, ${ }^{8}$ the result was symptomatic of a high value of $B$. This was judged to be unrealistic in the light of intuitive experience and the new data reported by Finlinson et $a .^{6}$ A response function curve having a more well-defined peak with higher values was required.

The problem was solved by changing some of the combustion constants in the model. This not only gave good steady-state and response function results, it was felt that the constituents of the results, for example, ranges of surface temperatures and condensed phase exothermicities, were now more consistent with earlier measurements (cf. Ref. 7 for a review). For convenience, the AP model is repeated here together with tables of input constants and steadystate results as follows.

The mass flux is given by an Arrhenius expression:

$$
m_{\mathrm{ox}}=\rho_{\mathrm{ox}} r_{\mathrm{ox}}=A_{\mathrm{ox}} \exp \left(-E_{\mathrm{ox}} / R_{u} T_{s}\right)
$$

The fraction of AP reacted in the gas phase is

$$
\beta_{p}=1-\frac{A_{s} \exp \left(-E_{s} / R_{u} T_{s}\right)}{m_{\mathrm{ox}}}
$$

The remainder of the AP reacts in the condensed phase to produce condensed-phase exothermicity. Here, the condensed-phase reactions are lumped at the surface (at $T=T_{s}$ ) but are numerically equivalent to the distributed reaction scheme of Ref. 7 over a finite depth in the condensed phase. The heat content of the adiabatic AP flame is

$$
Q_{F}=c_{g}\left(T_{\mathrm{ox}}-298\right)-c_{s}\left(T_{0}-298\right)+\Delta H_{g}
$$

The net surface heat release is

$$
Q_{L}=\beta_{p}\left(\Delta H_{\mathrm{ev}}-\Delta H_{g}\right)-\left(1-\beta_{p}\right) Q_{F}
$$

Thus, the net heat release in the AP flame is

$$
Q_{\mathrm{ox}}=Q_{L}+Q_{F}=\beta_{p}\left[\Delta H_{\mathrm{ev}}+c_{g}\left(T_{\mathrm{ox}}-298\right)\right]
$$

Equations (3-5) state that a portion of the AP gasifies $\left(\beta_{p}\right)$ and reacts in the gas phase to yield $Q_{\mathrm{ox}}$, and a portion reacts in the condensed phase $\left(1-\beta_{p}\right)$ to yield a net $Q_{L}$. What does not react in the condensed phase reacts in the gas phase. $Q_{F}$ is the total heat content. ( $Q_{L}$ is negative if exothermic in the sign convention used.) The various heats involved are defined in the Nomenclature.

The flame standoff distance is expressed as a flame sheet model, which is a well-known way of representing the gas phase. ${ }^{9}$ This also provides the temperature distribution in the gas for use in the steady-state energy balance and later as a boundary condition in the unsteady analysis. The expression here, like Eq. (2), is made numerically equivalent to the distributed reaction model used in Ref. 7:

$$
X_{\mathrm{ox}}^{*}=\frac{m_{\mathrm{ox}}}{A_{\mathrm{AP}} p^{2} \exp \left(-E_{\mathrm{AP}} / R_{u} T_{\mathrm{ox}}\right)}
$$

The dimensionless standoff is

$$
\xi_{\mathrm{ox}}=\left(c_{g} / k_{g}\right) m_{\mathrm{ox}} X_{\mathrm{ox}}^{*}
$$

Finally, the heat balance at the surface is written as

$$
T_{s}=T_{0}-\frac{Q_{L}+\Delta H_{s}}{c_{s}}+\frac{Q_{\mathrm{ox}} \exp \left(-\xi_{\mathrm{ox}}\right)}{c_{s}}
$$

The procedure is to solve for $T_{s}$ by iteration, which gives the burning rate. The input constants were originally taken from Ref. 2 and revised as a result of this work. The values used are given in Table 1 and the results of computations in Table 2. Various burn rate data for AP with results of past computations were published previously.,

The model continues to be in good agreement with the burning rate data, but the adjustments now enable much better computation of response function behavior as well. Also, the range of surface temperatures computed is now broader, and the fraction reacted in the condensed phase is now greater (yielding a more exothermic

\begin{tabular}{|c|c|}
\hline Parameter & Value \\
\hline$\rho_{\mathrm{ox}}, \mathrm{g} / \mathrm{cm}^{3}$ & 1.95 \\
\hline$A_{\mathrm{ox}}, \mathrm{g} /\left(\mathrm{cm}^{2} \mathrm{~s}\right)$ & $1.5665 \times 10^{8}$ \\
\hline$E_{\mathrm{ox}}, \mathrm{cal} / \mathrm{mole}$ & 32,000 \\
\hline$A_{s}, \mathrm{~g} /\left(\mathrm{cm}^{2} \mathrm{~s}\right)$ & $2.5004 \times 10^{7}$ \\
\hline$E_{s}, \mathrm{cal} / \mathrm{mole}$ & 30,000 \\
\hline$\Delta H_{\mathrm{ev}}, \mathrm{cal} / \mathrm{g}$ & 526.5 \\
\hline$\Delta H_{s}, \mathrm{cal} / \mathrm{g}$ & 138.5 \\
\hline$\Delta H_{g}, \mathrm{cal} / \mathrm{g}$ & $142.59^{\mathrm{a}}$ \\
\hline$c_{s}, \mathrm{cal} /(\mathrm{gK})$ & $0.3903^{\mathrm{a}}$ \\
\hline$c_{g}, \mathrm{cal} /(\mathrm{gK})$ & $0.2852^{\mathrm{a}}$ \\
\hline$T_{\mathrm{ox}}, \mathrm{K}$ & $1404.3^{\mathrm{a}}$ \\
\hline$A_{\mathrm{AP}}, \mathrm{g} /\left(\mathrm{atm}^{2} \mathrm{~cm}^{3} \mathrm{~s}\right)$ & 54,543 \\
\hline$E_{\mathrm{AP}}, \mathrm{cal} / \mathrm{mole}$ & 30,000 \\
\hline$k_{g}, \mathrm{cal} /(\mathrm{cm} \mathrm{sK})$ & 0.000515 \\
\hline
\end{tabular}
condensed phase), which are more consistent with the data ${ }^{7,10}$ than the Ref. 2 model in those respects. The flame heights are similar to the previous values, and so these changes do not significantly affect the competing flame process computed in the composite propellant model. The pressure exponent is roughly constant at 0.92 .

Table 1 Values of input constants for the AP model

Table 2 Computed steady-state results for the AP model

\begin{tabular}{lcccccc}
\hline \hline $\begin{array}{l}\text { Pressure, } \\
\text { atm }\end{array}$ & $\begin{array}{c}\text { Burn rate, } \\
\mathrm{cm} / \mathrm{s}\end{array}$ & $\begin{array}{c}\text { Surface } \\
\text { temperature, } \mathrm{K}\end{array}$ & $\beta_{p}$ & $\begin{array}{c}\text { Flame } \\
\text { height, } \mu \mathrm{m}\end{array}$ & $\begin{array}{c}Q_{L}{ }^{\mathrm{a}}{ }^{\mathrm{c}} \mathrm{c} / \mathrm{g} \\
\mathrm{c}^{2}\end{array}$ & $\begin{array}{c}Q_{\mathrm{ox}}, \\
\mathrm{cal} / \mathrm{g}\end{array}$ \\
\hline 21.5 & 0.279 & 826.7 & 0.461 & 10.99 & -65.75 & 386.29 \\
38.3 & 0.477 & 850.2 & 0.479 & 5.67 & -53.01 & 402.10 \\
68.0 & 0.812 & 874.8 & 0.496 & 2.93 & -40.86 & 417.32 \\
121.1 & 1.379 & 900.7 & 0.512 & 1.50 & -29.19 & 432.09 \\
215.0 & 2.322 & 927.7 & 0.528 & 0.77 & -18.15 & 446.24 \\
\hline \hline
\end{tabular}

${ }^{a}$ Negative values denote an exothermic condensed phase. 
Such a high value of exponent would be expected to magnify the combustion response to pressure perturbations.

Although the model can compute the steady-state burn rates at lower pressures, in reality AP does not burn below about 20 atm. A possible mechanism for this is excessive condensed-phaseexothermicity. When the major portion of the heat feedback to the burning surface is due to condensed-phase exothermicity, rather than from the gas-phase flame, the combustion approachesdynamic instability and an inability to sustain itself (cf., e.g., Ref. 11). Table 2 shows that condensed phase exothermicity is becoming more important and the gas phase less important (greater flame standoff, lower $Q_{\text {ox }}$ ) with decreasing pressure. Of course, in a composite propellant, the strong diffusion flame can sustain the dynamic stability of AP combustion down to much lower pressures.

\section{Validation of Numerical Method}

\section{Numerical Method}

Nonsteady combustion analysis requires solution of the transient heat conduction equation in the solid phase. The thermal wave response in the solid is the important time lag mechanism; a quasisteady gas is assumed, valid at the axial-mode frequencies of interest. Note that in a two-component system such as a composite propellant, a double iteration is required for AP and binder because of their differing thermal wave properties; they are coupled through the flame processes. For the AP alone, as an initial step in the modeling, only the AP is involved.

The Crank-Nicholson numerical scheme is employed, with coordinate stretching to obtain a finer grid near the solid surface. The boundary condition in the deep solid is the bulk temperature. The value of surface temperature is guessed in the iteration at each time step and used in the surface boundary condition for the heat conduction equation. Heat flux at the surface is calculated using the temperature gradient at the surface $[\partial \theta / \partial z]_{\text {solid }}$ and matched to the heat flux from the gas phase $[\partial \theta / \partial z]_{\text {gas }}$ using the energy balance. These are dimensionless quantities. The discrepancy between the obtained values, $\delta$, is set to be less than $10^{-9}$ as the convergence criterion. For the iterations, new guesses for dimensionless surface temperature $\theta_{s}$ are given by

$$
\left[\theta_{s}\right]_{i+1}=\left[\theta_{s}\right]_{i}-\frac{\delta_{i}\left(\left[\theta_{s}\right]_{i}-\left[\theta_{s}\right]_{i-1}\right)}{\delta_{i}-\delta_{i-1}}
$$

where $i+1, i$, and $i-1$ denote three consecutive iterations. There are 201 mesh points, and the dimensionless time step is 0.001 . A relativelyhigh accuracy of computationis needed because Arrhenius kinetics yield small changes in $\theta_{s}$.

Linearized theory in the numerical scheme is approximated by inputting sine wave perturbations of varying dimensionless frequencies $\Omega$ and with a dimensionlesspressure amplitude $(P-1)$ of 0.01 . Larger amplitudes are used to evaluate differences between linear and nonlinear behavior. It is also possible to input sine waves having more than one frequency and other nonlinear waveforms such as sawtooths. Output includes dimensionless instantaneous burn rate $R$; other variables may also be output for debug or diagnostic studies. Convergence of the numerical scheme has been very rapid so that it has been practical to divide the range of $\Omega$ into 150 increments for the computations of response function curves. Response functions, real and imaginary parts, are computed internally from the oscillations and phase relationships following equilibration. There is a preliminary startup transient because the initial condition is the steady-state solution.

\section{Validation with Linear Theory}

It is recommended that analytical studies of this type begin by validating the numerical method with the classical linear response function theory. The theory gives the following, well-known, closedform expression for the response function ${ }^{8}$ :

$$
R_{p}=\frac{n A B}{A B-(1+A)+A / \lambda+\lambda}
$$

The numerical method was checked by replacing the AP model with the Levine and Culick model,${ }^{12}$ which is known to reduce to the classical theory in the linear limit. This model gives the following linearized boundary condition at the solid surface ${ }^{8,12}$ :

$$
\left[\frac{\partial \theta}{\partial z}\right]_{\mathrm{gas}}=1+n B(P-1)+\left(1-B+\frac{1}{A}\right)(R-1)
$$

with $(R-1)=A\left(\theta_{s}-1\right)$.

Published ranges of combustion constants and experience in applying this method to AP propellantshave provided a feel for reasonable values of $A$ and $B$. $A$ should range from about 6 to 12 and $B$ from about 0.6 to 1.2 . Increasing $A$ increases the resonant (peak) value of $R_{p}$ and the peak response $\Omega$. Reducing $B$ increases and sharpens the resonant peak response. Thus, combinations of higher $A$ and lower $B$ yield stronger responses. Validations were performed with $A=6$ and $B=1$ (relatively weak response), $A=8.5$ and $B=0.8$, and $A=11$ and $B=0.7$ (relatively strong response). Results for the real part of $R_{p}$ shown in Figs. 1a-1b show precise agreement between the numerical method and the exact mathematical solution.

\section{Validation with Nonlinear Boundary Condition}

Another approach is to use the general nonlinear form of the Levine and Culick mode ${ }^{12}$ and make computations in the limit of small perturbations. Then, with the nonlinear boundary condition, it is possible to increase the pressure amplitudes to explore departures from the linear behavior. The nonlinear Levine and Culick boundary condition at the propellant surface ${ }^{12}$ is

$$
\begin{aligned}
& {\left[\frac{\partial \theta}{\partial z}\right]_{\text {gas }}=\frac{P^{2 n}}{R}\left\{1+\frac{1}{1-\theta_{0}}\left[\frac{1}{1-(n / A)\left(1-\theta_{0}\right) \ln P}-1\right]\right.} \\
& \left.-\left[1-0.5\left(B-\frac{1}{A}\right)\right]\left(1-\frac{R^{2}}{P^{2 n}}\right)\right\}
\end{aligned}
$$

Pressure amplitudes of 1,5 , and $10 \%$ were used for each of the three combinations of $A$ and $B$. Results are shown, together with results from Eq. (10), in Figs. 2a-2c. The agreement with the linear theory for $1 \%$ perturbations is excellent in the cases of weak and moderate response, but there is a small difference in the case of strong response. This shows that $1 \%$ cannot always be taken for granted as a small perturbation for linearized analyses. On the other hand, for the cases of weak and moderate response, even a $10 \%$ pressure amplitude does not produce significant changes from the linear behavior. For the case of strong response, a 5\% amplitude produces a significant but not large departure about the resonant frequency; it requires a $10 \%$ amplitude to see a large effect.

The work of Levine and Baum ${ }^{1}$ and a recent approximate nonlinear mathematical analysis by Culick ${ }^{13}$ have shown that nonlinear pressure coupling alone cannot describe features of nonlinear instability observed in motors. In each case, it was concluded that a response to crossflow perturbations with a threshold (analogous to the erosive burning threshold) is required. That is why turbulence modeling is an important part of any combustion modeling that will be coupled to modeling a flowfield analysis. Figure 2 appears to confirm that realistic pressure disturbances (less than 5\%) alone are generally inadequate to evoke nonlinear behavior.

\section{AP Model Results}

The nonlinear surface boundary condition for the AP model, derived in the manner shown by Levine and Culick ${ }^{12}$ with their model, is

$$
\left[\frac{\partial \theta}{\partial z}\right]_{\text {gas }}=R\left\lfloor-H_{c}+H_{\mathrm{ox}} \exp \left(-\xi_{\mathrm{ox}}\right)\right\rfloor
$$

where $H_{c}=\left(Q_{L}+\Delta H_{s}\right) / c_{s}\left(\bar{T}_{s}-T_{0}\right)$ and $H_{\mathrm{ox}}=Q_{\mathrm{ox}} / c_{s}\left(\bar{T}_{s}-T_{0}\right)$.

Note that the heat release and flame height terms are functions of the instantaneous burn rate and pressure (refer back to the model) computed as part of the numerical scheme. The heat release terms are also a function of the phase in the cycle because of the thermal response of the condensed phase..$^{8,12}$

Results for $1 \%$ pressure amplitude at each of three pressures are compared with the Finlinson et al. ${ }^{6}$ data in Figs. 3a-3c. In making 


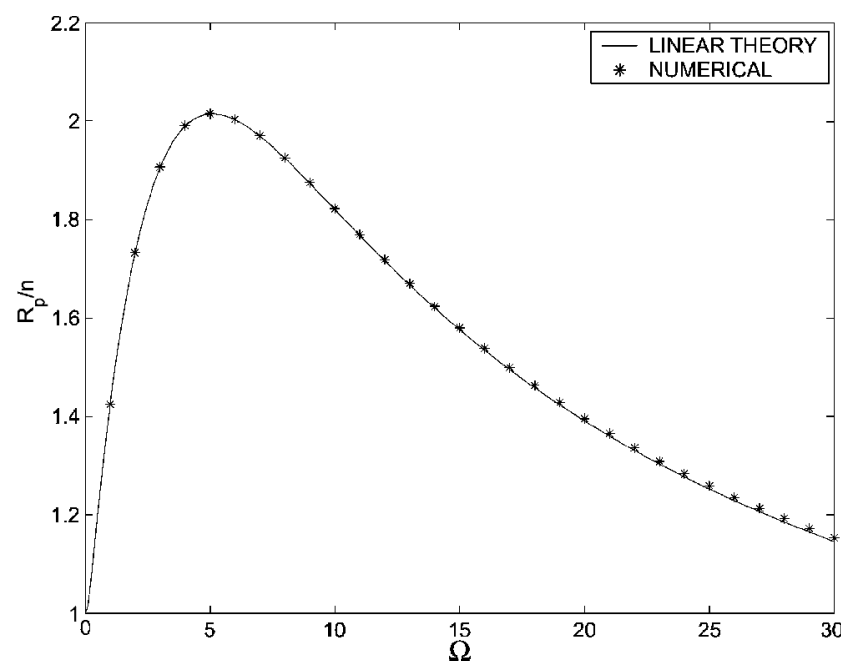

a) $A=6$ and $B=1$

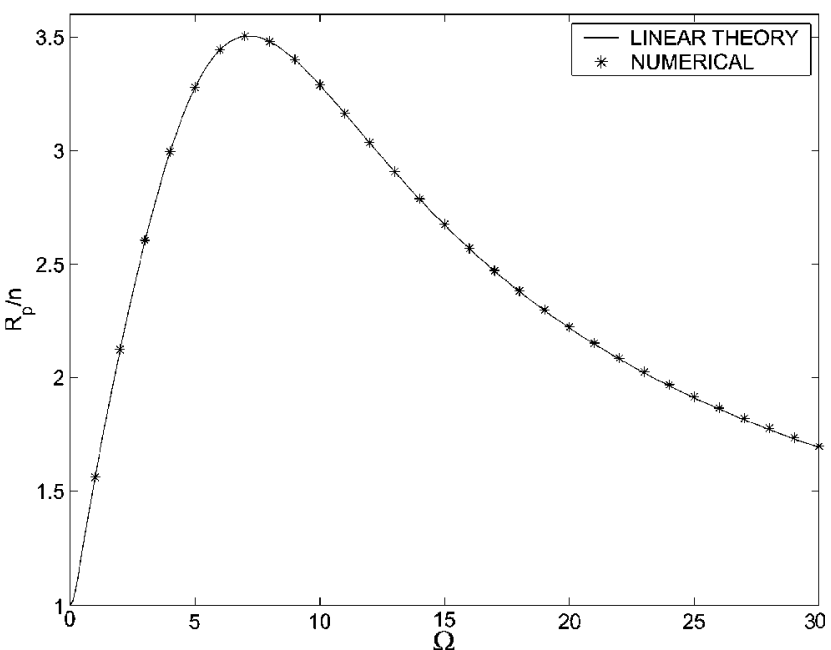

b) $A=8.5$ and $B=0.8$

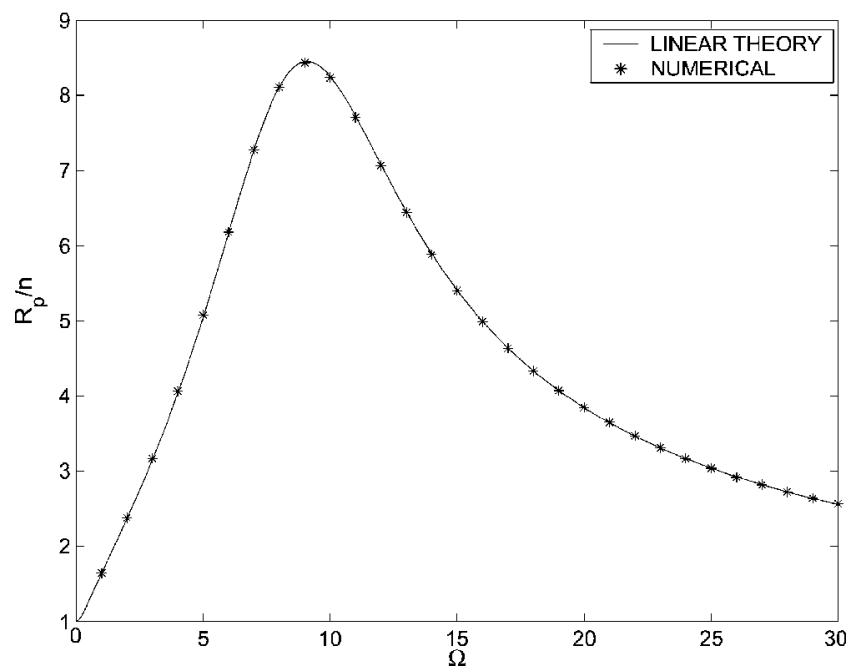

c) $A=11$ and $B=0.7$

Fig. 1 Comparisons of numerical and mathematical solutions for linearized pressure-coupled response.

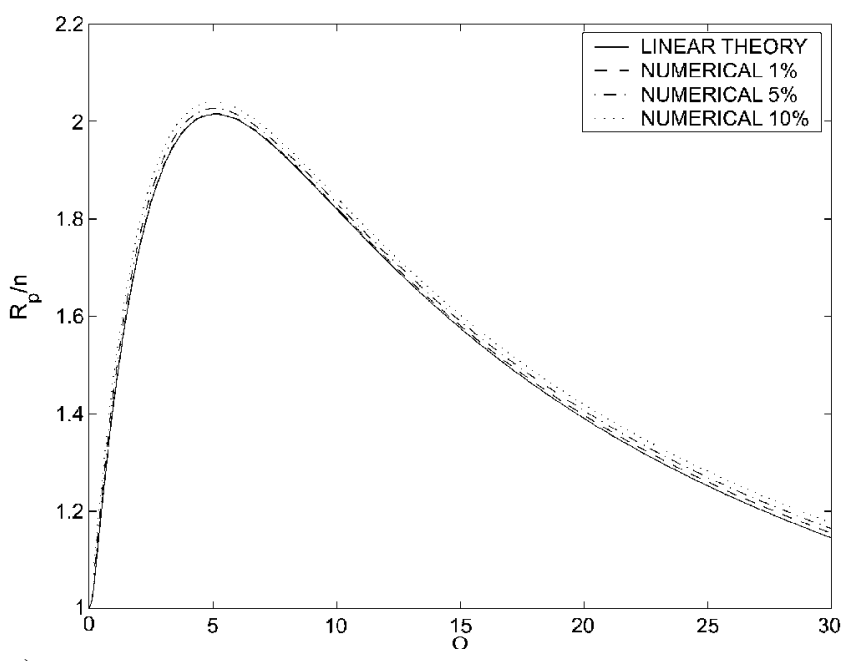

a) $A=6$ and $B=1$

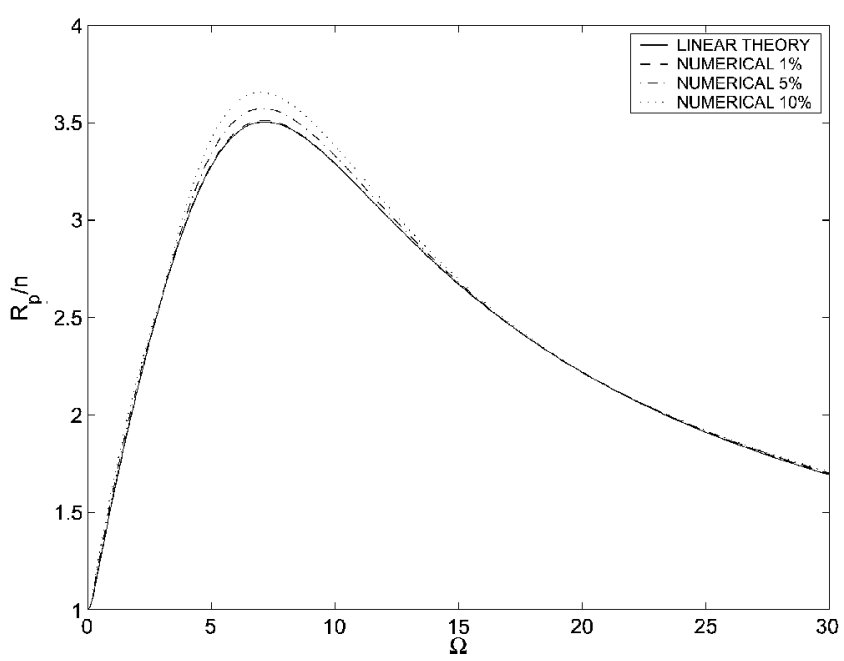

b) $A=8.5$ and $B=0.8$

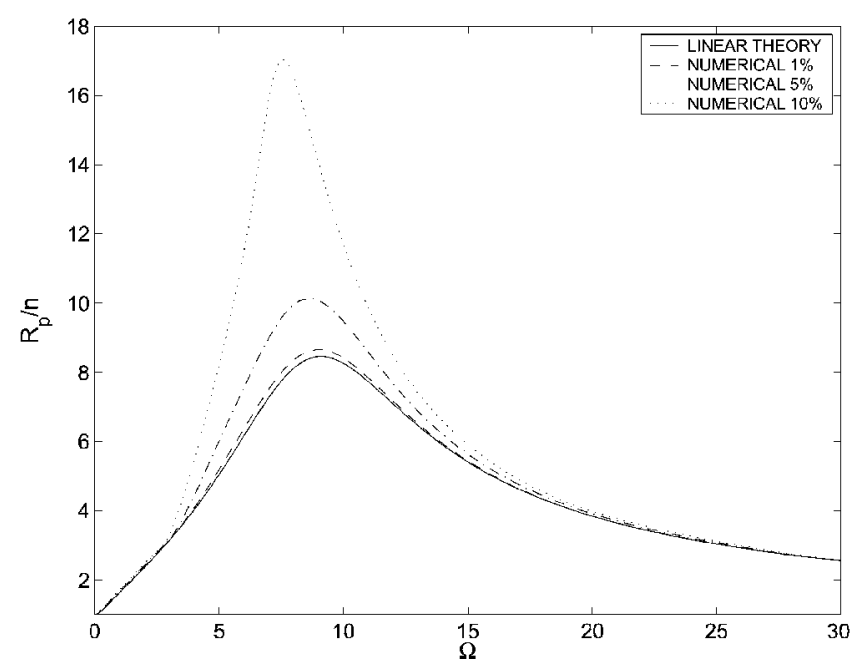

c) $A=11$ and $B=0.7$

Fig. 2 Effect of oscillatory pressure amplitude on the combustion response, nonlinear boundary condition. 


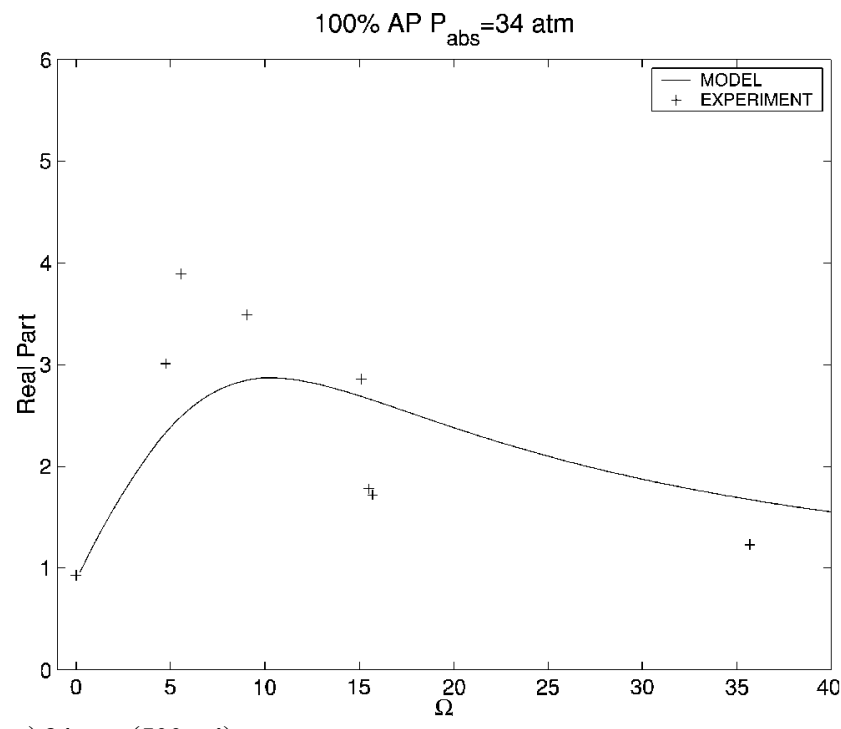

a) $34 \mathrm{~atm}(500 \mathrm{psi})$

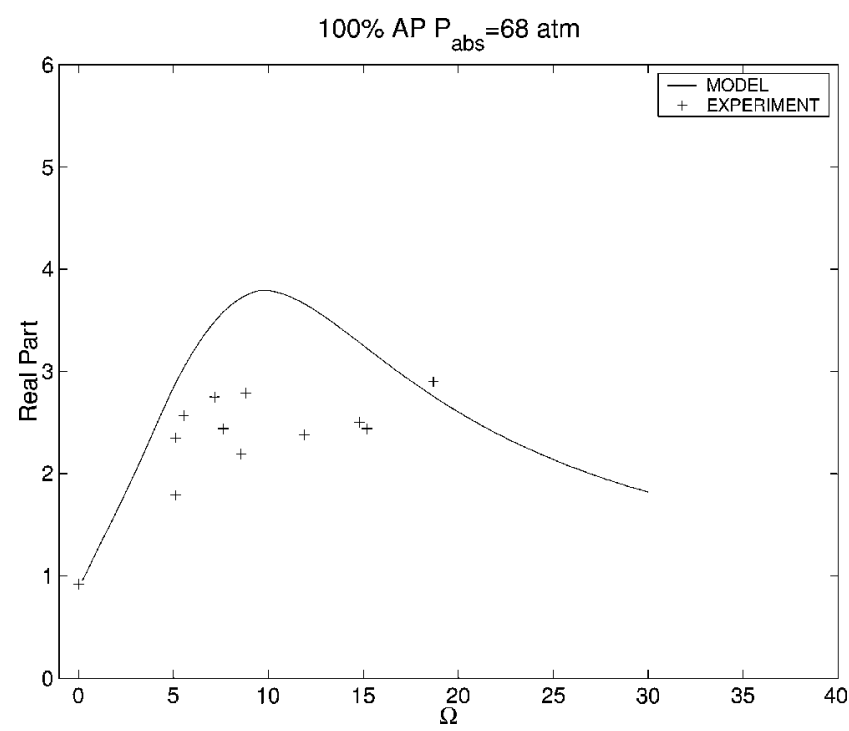

b) $68 \mathrm{~atm}(1000 \mathrm{psi})$

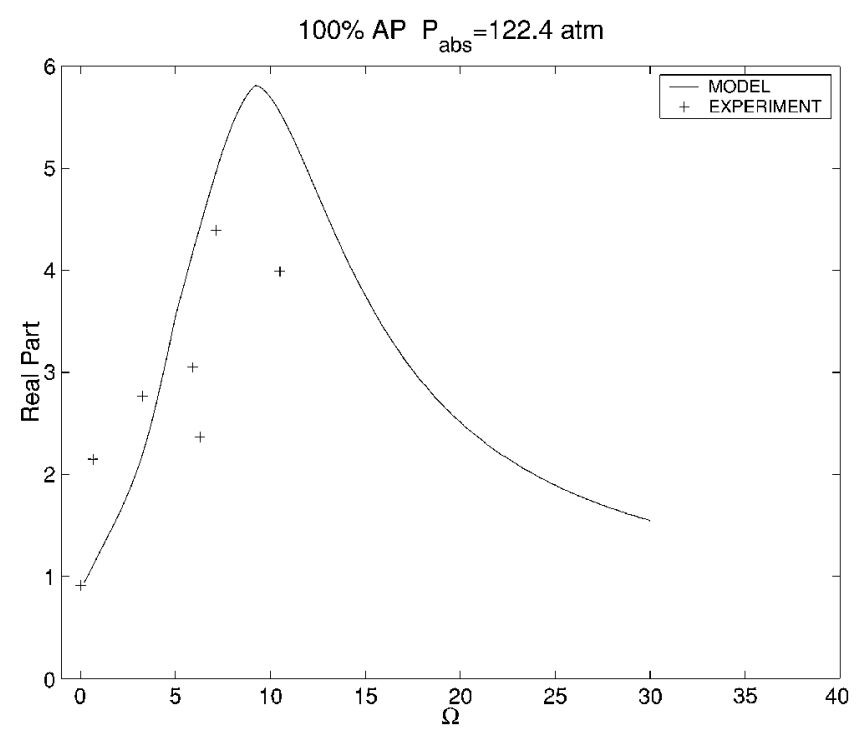

c) $122.4 \mathrm{~atm}(1800 \mathrm{psi})$

Fig. 3 Comparisons of pressure-coupled response functions computed from the AP model with experimental data. the comparison, published values of Ref. 6 of $\Omega$ were adjusted for consistency in the values of thermal diffusivity used to normalize the test frequencies. It is generally agreed that the value should be an average over the temperature range in the solid-phase thermal wave, as determined from Ref. 10. Measured response functions less than 1 were omitted as probably erroneous. The experimental pulsed-T-burner technique measures a small differencebetween two large numbers and is suspect for excessiveerror when that difference becomes very small. Even good data are known to exhibit significant scatter due to variabilities that influence the outcome of the test.

On the whole, the model is in reasonable agreement with the data. The data scatter shown here is what is normally acceptable from T-burners, and there are enough data for curve fits to evince trends with frequency and pressure within that accuracy. ${ }^{6}$ The positions of the analytical curves are within or comparable to the data scatter. The peak responseis underpredictedat $34 \mathrm{~atm}$, is overpredictedat $68 \mathrm{~atm}$, and is possibly overpredicted at $122 \mathrm{~atm}$. The model is predicting an increase in the peak response with increasing pressure, whereas the data suggest that the peak response goes through a minimum at an intermediate pressure. The predictions at high pressure are interesting in that Finlinson et al. did not expect the high values measured at that pressure. ${ }^{6}$

If a minimum combustion response at an intermediate pressure is a real effect, not just data scatter, one can only speculate on the reason for that at this time. Some speculation will be included in the discussion of the controlling mechanisms within the model that follows.

\section{Discussions of Mechanisms and Properties of the Model Peakedness of Response Function Curves}

It was noted earlier that response functions predicted with the AP model in its originalform were unsatisfactory,necessitating changes in some of the combustion constants to achieve good predictions of both steady-stateburn rates and response function behavior. A study of the boundary condition revealed the factors influencing whether a computed response function curve would be flat or able to have sharper peaks, and this study led to the changes that were made.

The key is in the relative behavior of the two heat release terms in the bracket of Eq. (13), namely, how $H_{c}$ and $\left[H_{\text {ox }} \exp \left(-\xi_{\text {ox }}\right)\right]$ change with instantaneousburning rate. Figure 4 is a plot of $[\partial \theta / \partial z]_{\text {gas }}$, the driving temperature gradient at the surface, vs imposed burning rates for two cases. The first case, showing a decrease in the gradient with increasing burn rate, typifies that which yields the computation of flat responsefunction curves. That is the result with the originalset of constants. The second case, showing an increase in the gradient with increasing burn rate, results in the computation of peaked response function curves. That is the result with the current set of constants. When the driving heat flux decreases with increasing burn rate (as in the first case), it is a stabilizing influence or effect. Correspondingly,

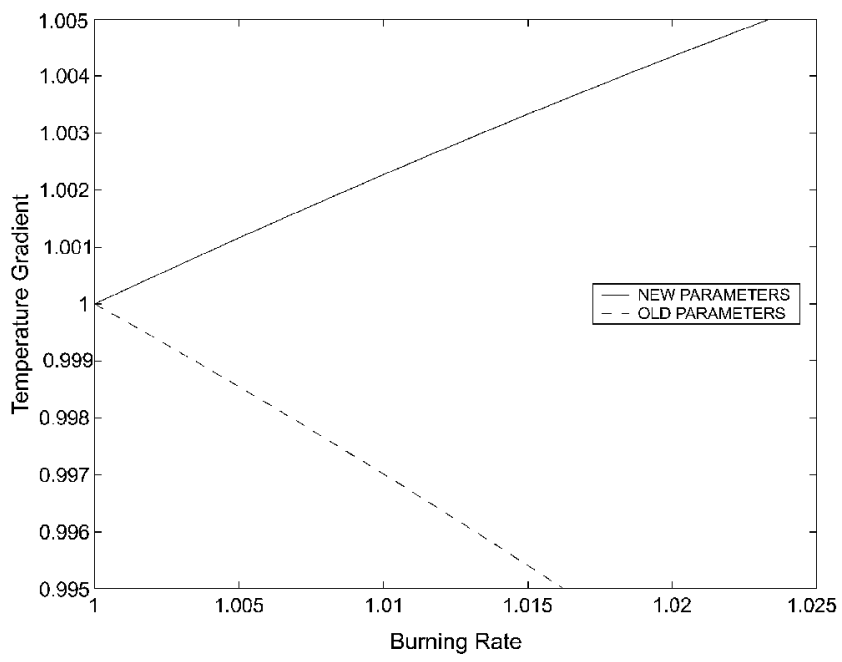

Fig. 4 Computed, instantaneous temperature gradients from the AP model surface boundary condition vs specified burning rates; original model (negative slope) and revised model (positive slope). 
when it increases with increasing burn rate (as in the second case), it is destabilizing.

$H_{c}$ is made up of the condensed phase heat release $Q_{L}$ (exothermic) and the latent heat $\Delta H_{s}$ (endothermic). $H_{\mathrm{ox}}$ is dimensionless $Q_{\text {ox }}$, the heat release in the flame, and the exponential in $\xi_{\text {ox }}$ (the dimensionless flame height) multiplies it to give the heat feedback from the flame. The gradient $[\partial \theta / \partial z]_{\mathrm{gas}}$ decreases with increasing burn rate when $H_{c}$ increases (becomes less exothermic or more endothermic) with burn rate to a greater extent than the heat feedback from the flame. The constants were changed to reverse this trend. To reduce the increase in $H_{c}$, either $H_{c}$ has to be a smaller number to begin with or it has to be less sensitive to burn rate. Both were accomplishedby making $Q_{L}$ more exothermic (reducing $\beta_{p}$ ) and by increasing the activation energy of the condensed phase reactions ( $E_{s}$ closer to $E_{\text {ox }}$ ). The flame kinetics was adjusted to maintain the correct steady-state burn rate behavior; the adjustment needed turned out to be small so the impact of the flame on the relative behavior of the terms was small.

This exercise has important implications for stability. Energetic materials do have exothermic condensed-phase reactions. It is desired, for stability, that these reactions be less exothermic in net magnitude and/or that they have low-activation energies. Studies of other ingredients (nitramines, nitrate esters, azido compounds, etc.) and future chemistry research into advanced propellant ingredients should keep these properties in mind. A mathematical criterion for improved stability can be specified as

$$
\left.\frac{\partial H_{c}}{\partial R}\right|_{P} /\left.\frac{\partial\left[H_{\mathrm{ox}} \exp \left(-\xi_{\mathrm{ox}}\right)\right]}{\partial R}\right|_{P}>1
$$

\section{$A$ and $B$ Parameters for the AP Model and Intrinsic Instability}

It was noted earlier that the $B$ parameter in the classical theory determines the extent of the peakednessin responsefunction curves. In view of the foregoing discussion, a mathematical analysis was conducted to derive expressions for $A$ and $B$ from the AP model to provide more insight into this mechanistic behavior. The expressions were obtained by linearizing the surface boundary condition, Eq. (13), and comparing the result with the classical formulation, Eq. (11). The results are

$$
\begin{aligned}
& A=\frac{E_{\mathrm{ox}}\left(\bar{T}_{s}-T_{0}\right)}{R \bar{T}_{s}^{2}} \\
& B=\bar{H}_{\mathrm{ox}}\left\{\begin{array}{c}
\left(1-\frac{E_{s}}{E_{\mathrm{ox}}}\right) \frac{\left(1-\bar{\beta}_{p}\right)}{\bar{\beta}_{p}}\left[1-\exp \left(-\bar{\xi}_{\mathrm{ox}}\right)\right] \\
+2 \bar{\xi}_{\mathrm{ox}} \exp \left(-\bar{\xi}_{\mathrm{ox}}\right)
\end{array}\right\}+\frac{1}{A}
\end{aligned}
$$

The expression for $A$ turns out to be the same as in the classical theory. ${ }^{8,12}$ It is in the expressionfor $B$ that the particular components of the AP model are reflected.

Figures 5 and 6 are plots of $A$ and $B$ over the pressure range of interest. It is observed that $A$ is roughly constant, decreasing from 12.4 at $20 \mathrm{~atm}$ to 11.9 at $130 \mathrm{~atm}$. Calculations above $130 \mathrm{~atm}$ are speculative because of a lack of data. Response function results are largely due to the changes in $B$, decreasing from 1.02 at 20 atm to 0.75 at $130 \mathrm{~atm}$. When Eq. (16) is referred to, $B$ decreases with increasing pressure because the terms $\left(1-\bar{\beta}_{p}\right) / \bar{\beta}_{p},\left[1-\exp \left(-\bar{\xi}_{\text {ox }}\right)\right]$, and $2 \bar{\xi}_{\text {ox }} \exp \left(-\bar{\xi}_{\text {ox }}\right)$ each decrease with increasing pressure as continuous functions. The energy term $\bar{H}_{\text {ox }}$ is roughly constant, as is $1 / A$.

This result does not explain the low-pressure deflagration limit of AP in terms of a theory of condensed-phase exothermicity, nor the apparent minimum in the peak response at 68 atm (namely, the measured peak response at 34 and 122 atm being greater than at $68 \mathrm{~atm}$ ) assuming that to be a real effect and not data scatter. To explore this further, it is instructive to examine $B$ under the limiting conditions of $\beta_{p}=1$ (all exothermic reactions in the gas phase) and $\beta_{p}=0$ (all in the condensed phase). For all reactions in the gas phase,

$$
\left.B\right|_{\beta_{p} \rightarrow 1}=\frac{2 \bar{\xi}_{\mathrm{ox}}\left[c_{g}\left(\bar{T}_{\mathrm{ox}}-298\right)+\Delta H_{\mathrm{ev}}\right]}{c_{s}\left(\bar{T}_{s}-T_{0}\right)}+\frac{1}{A}
$$

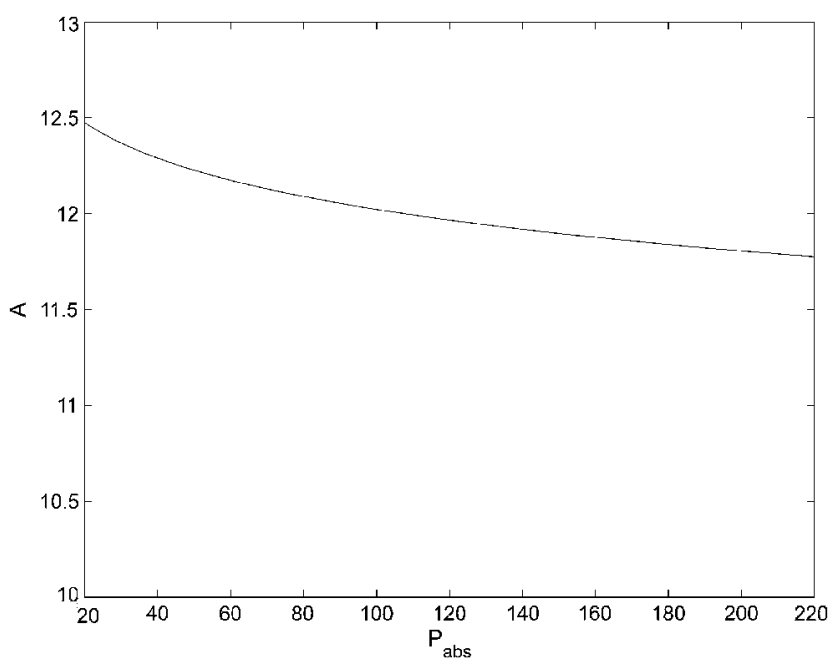

Fig. 5 Plot of $\boldsymbol{A}$ from the AP model vs steady-state pressure (atm).

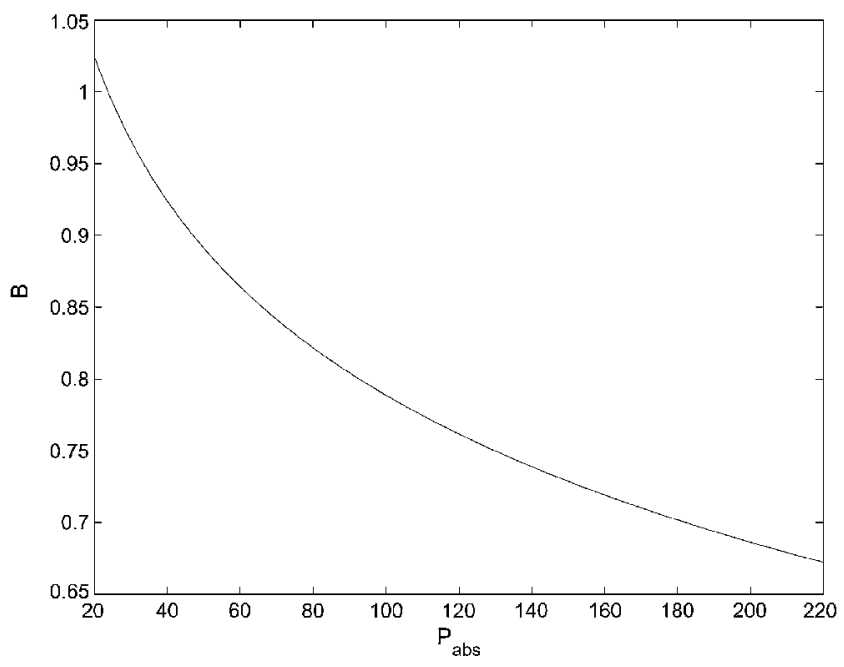

Fig. 6 Plot of $B$ from the AP model vs steady-state pressure (atm).

With the parametrics of this model, approaching this condition requires enormous pressures wherein $\bar{\xi}_{\text {ox }}$ becomes vanishingly small. Compared to the changes in $\bar{\xi}_{\text {ox }}$ with pressure, the ratio that it multiplies and the term $1 / A$ are relatively constant with pressure. The value of $\bar{\xi}_{\text {ox }}$ at $215 \mathrm{~atm}$ is about 0.2 . When $\bar{\xi}_{\text {ox }}$ is taken to be 0.02 and $A=11.5$, the value of $B$ is computed to be about 0.15 . Such a low value of $B$ raises a question about intrinsic instability at very high pressures and argues for more response function data above $122 \mathrm{~atm}$. (The Finlinson et al. ${ }^{6}$ apparatus can go to $270 \mathrm{~atm}$.) Very strong responses would be expected at the higher pressures, which could have implications for propellants because the AP flame controls the propellant behavior at high pressures. ${ }^{2}$

For all reactions in the condensed phase (no flame or infinite flame standoff distance),

$$
\left.B\right|_{\beta_{p} \rightarrow 0}=\frac{\left(1-E_{s} / E_{\mathrm{ox}}\right)\left[c_{g}\left(\bar{T}_{\mathrm{ox}}-298\right)+\Delta H_{\mathrm{ev}}\right]}{c_{s}\left(\bar{T}_{s}-T_{0}\right)}+\frac{1}{A}
$$

With the parametrics of this model, approaching this condition requires essentially zero pressure. Forcing $\beta_{p}$ to zero yields a value of $B$ of about 0.2 , which also raises a question about intrinsic instability at very low pressures.

The intrinsic instability boundary arises where the denominator of Eq. (10) vanishes. The following criterion has been derived to describe this condition ${ }^{8}$ :

$$
\frac{A(1-B)^{2}}{(1+B)}>1
$$

Note that intrinsic instability can arise from high as well as low values of $B$. As already discussed, high $B$ implies that heat feedback 
from the flame has the lesser sensitivity to burn rate, low $B$ implies that condensed-phaseexothermicity has the lesser sensitivity to burn rate. The case of low $B$ with the associated limiting conditions on $\beta_{p}$ is of more interest for discussion.

Figure 7 is a plot of this criterion for the AP model over a very wide pressure range. There is a high-pressure branch and a lowpressure branch, the reversal occurring near $20 \mathrm{~atm}$. That this reversal occurs near the low-pressure deflagration limit of AP is just a coincidence and should not be given any significance. On the highpressure branch, intrinsic instability is not yet achieved at $220 \mathrm{~atm}$, but the trend is to achieve it perhaps by $300 \mathrm{~atm}$. At that pressure, the flame is virtually on the surface and that appears to be a highly destabilizing influence. Lacking data, not much more can be said about it. On the low-pressure branch, the criterion is met at about $1 \mathrm{~atm}$.

When terms of the $\beta_{p} \rightarrow 0$ limit are used rather than the actual parametrics of the model, the ratio in Eq. (18) with $B=0.2$ would be about 7 at that limit. At that limit, the reactions are again taking place on the surface but from the solid side rather than the gas. Thus, the criterion comes to full circle, with gas-phase reactions on the surface dominating at high pressure (perhaps a very high pressure) and condensed-phasereactions on the surface dominating at low pressure (maybe not too low a pressure). Either situation of reactions on the surface, the full brunt of the heat release being tied to $\theta_{s}$, is highly destabilizing.

One can now speculate that response function data at pressures not far from the actual low-pressure limit of 20 atm (like $34 \mathrm{~atm}$ )

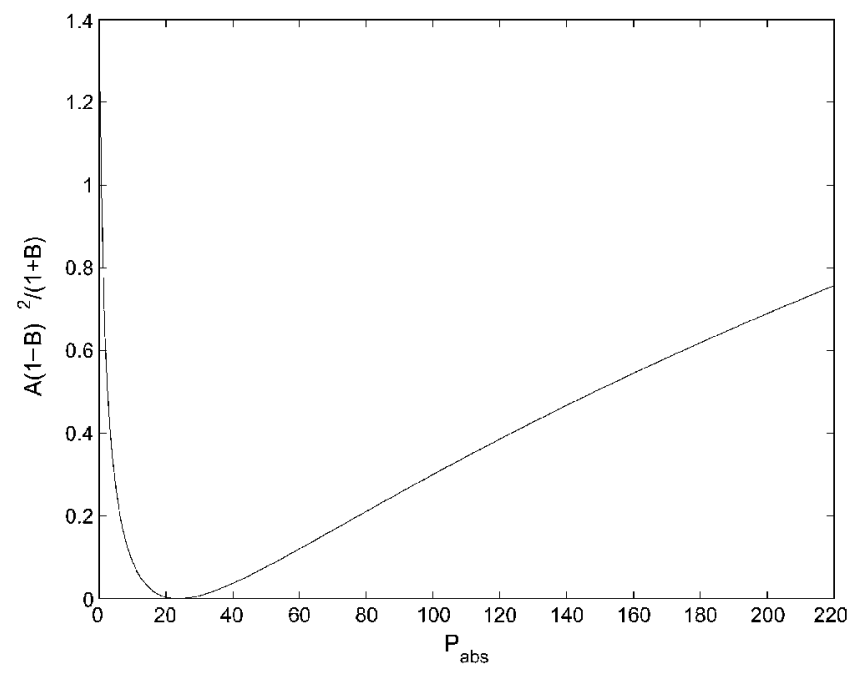

Fig. 7 Intrinsic instability criterion for the AP model vs steady-state pressure.
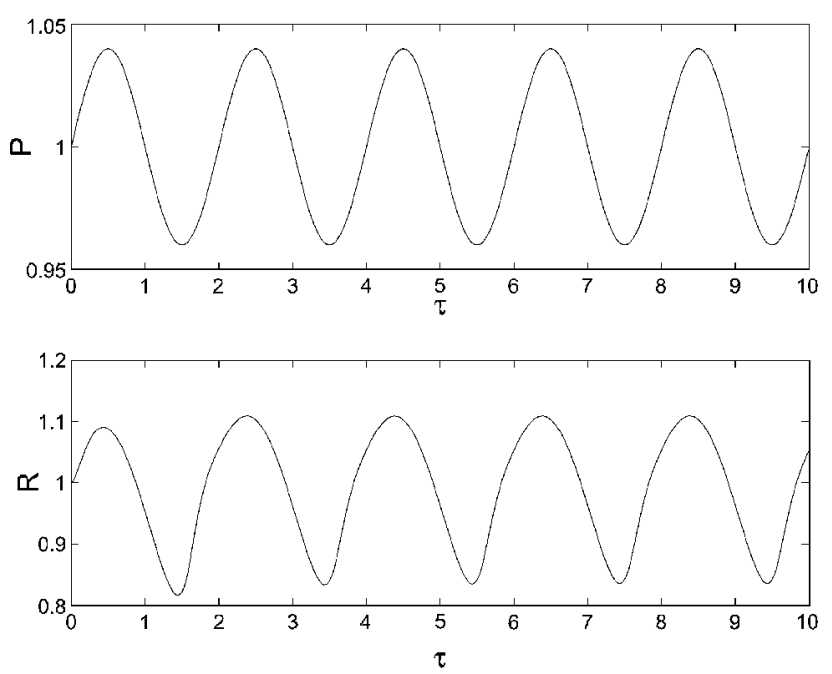

Fig. 8 Combustion response of the AP model to an imposed sinusoidal wave: $4 \%$ amplitude at $\Omega=5,68 \mathrm{~atm}$. can yield high values and data at high pressures approaching intrinsic instability can also yield high values, with lower values at intermediate pressures. More data, at a pressure such as $24 \mathrm{~atm}$, as well as at, for example, $240 \mathrm{~atm}$, are recommended. The parametrics of the model could be adjusted to shift the steep curve at low pressure in Fig. 7 so that intrinsic instability is predicted to occur at $20 \mathrm{~atm}$. However, the possibility that the limit at $20 \mathrm{~atm}$ is caused by a change in mechanism (a discontinuity at low temperature) should not be dismissed, and it remains possible that scatter in the response function data are obscuring the correct trend, such that the current parametrics are satisfactory.

\section{Nonlinear Combustion Response}

Figures 8-10 show representative nonlinear computations with the AP model for three kinds of imposed pressure waves at a mean pressure of $68 \mathrm{~atm}$. The first is a sine wave with $P=1.04$ at $\Omega=5$. The second superposes a harmonic having $P=1.02$ to simulate a nonlinear waveform. The third is a sawtooth with $P=1.04$ at the same fundamental frequency to simulate high harmonic content. These computations also served to check the general operation of the code.

The response to the sinusoidal oscillation produces a small but noticeable distortion in the sine wave. There is a phase lead in the combustion response, about $5 \%$ of the period, which is too small to notice. These computations begin at the steady-state condition (zero time), showing that the transient to equilibration is relatively short. The response to the harmonic wave reflects the stronger response at
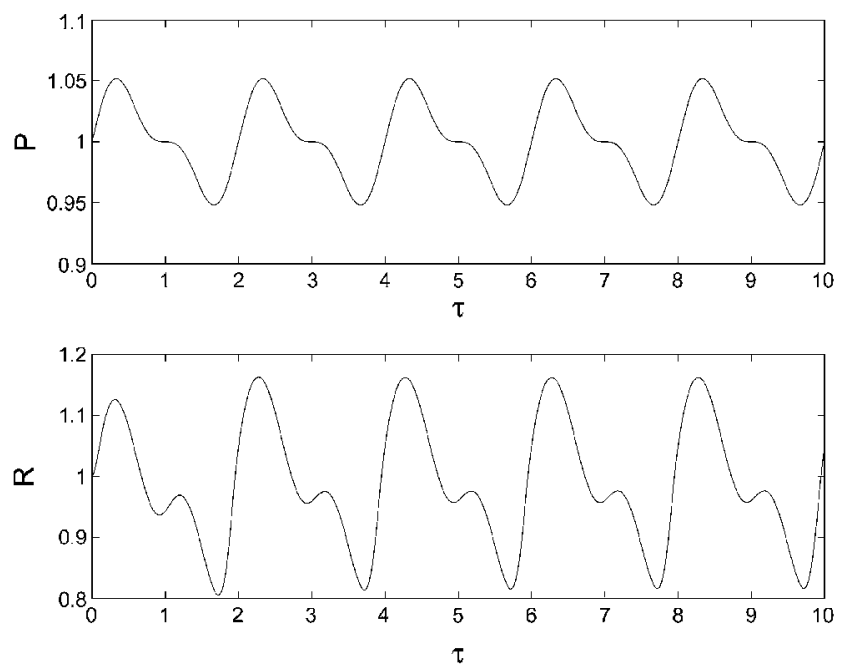

Fig. 9 Combustion response of the AP model to an imposed harmonic waveform: $4 \%$ amplitude at $\Omega=5$ and $2 \%$ amplitude at $\Omega=10,68 \mathrm{~atm}$.
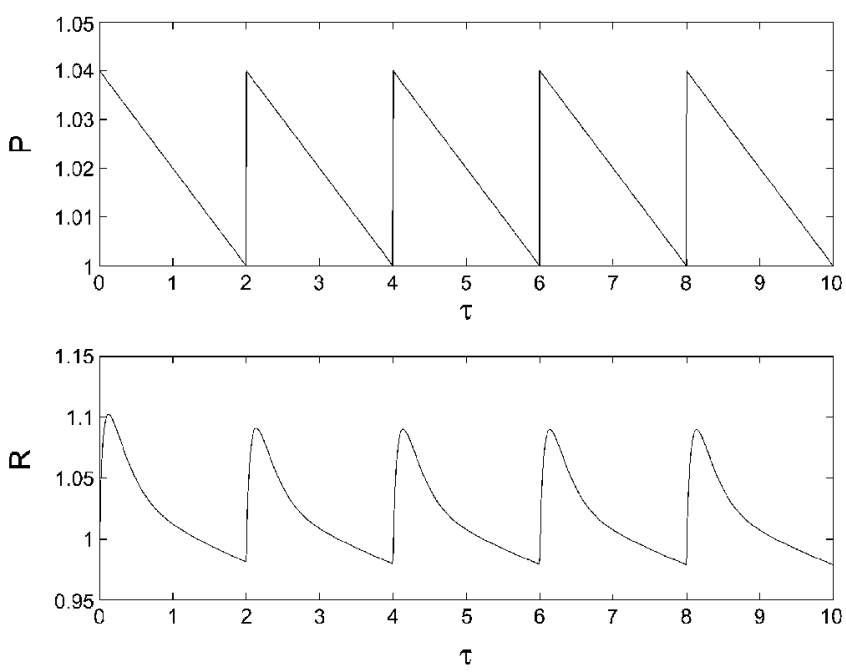

Fig. 10 Combustion response of the AP model to an imposed sawtooth wave: $4 \%$ amplitude at $\Omega=5,68 \mathrm{~atm}$. 
the higher frequency so that the harmonic blip is sharpened. There is a small phase lead at the lower frequency and a small phase lag at the higher frequency. The response to the sawtooth is interesting in that the response is smaller in magnitude and kept more in phase with the pressure oscillations. The response to the sawtooth is more rapid at the start of each fluctuation, up or down, and then slows toward the end of each fluctuation. The smaller magnitude of the response reflects the high harmonic content of the sawtooth wave. For each of these three waveforms, there is not a significant qualitative difference between the combustion response wave and the pressure wave. On the other hand, the response to a velocity wave can produce significant qualitative differences, especially if an acoustic erosivity threshold is crossed. ${ }^{14}$

Figures 11 and 12 show responses to $20 \%$ pressure oscillations, representative of fully developed instabilities in motors. The response to the sawtooth is qualitatively similar to that for the smaller disturbance. However, the response to the sine wave is very different. With the parametrics of this model, an extinguishment is predicted to occur upon the first pressure decay. This nonlinear effect takes place because the surface temperature cannotrecoverfrom the rapid burnoff (loss) of the steepened temperature gradient at the surface while the thermal profile is adjusting. It occurs more readily for the sine wave because the pressure falls to a lower value at a higher rate of pressure decay, and the response is more focused at the single frequency. This is a very interesting and perhaps significant pressure-coupled effect with the AP as a monopropellant, which could have implications for the stability of propellants, al-
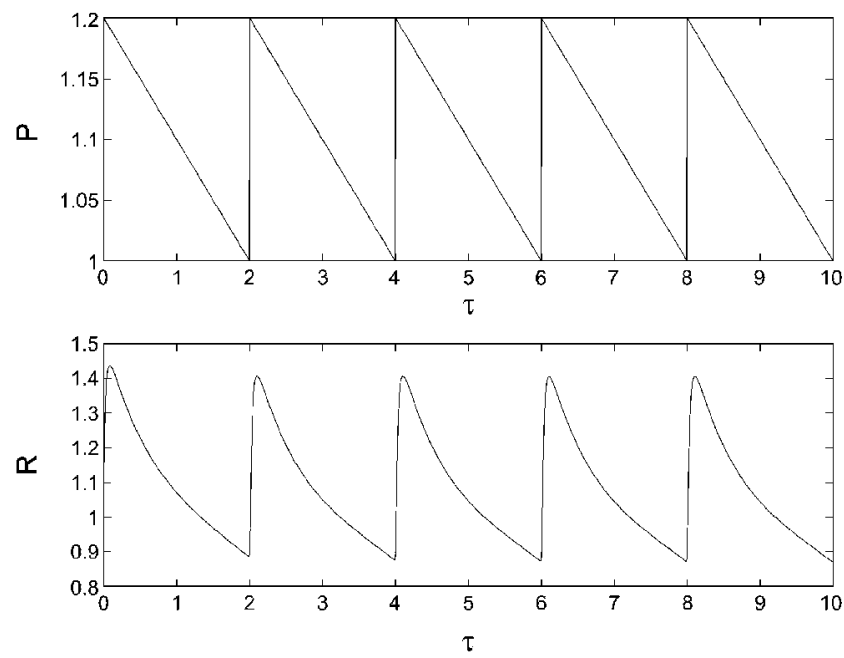

Fig. 11 Combustion response of the AP model to an imposed sawtooth wave: $20 \%$ amplitude at $\Omega=5,68 \mathrm{~atm}$.
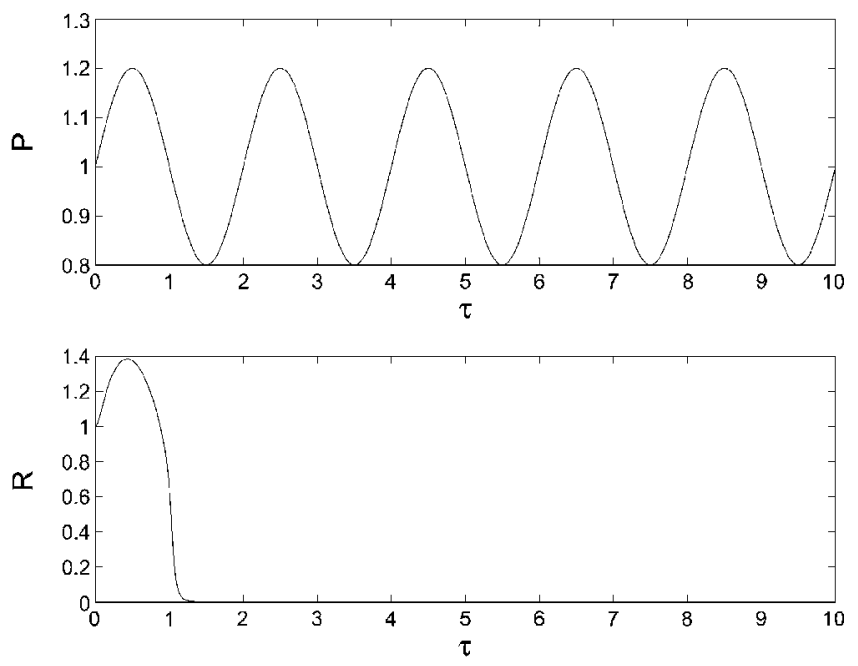

Fig. 12 Combustion response of the AP model to an imposed sinusoidal wave: $20 \%$ amplitude at $\Omega=5,68$ atm. though the presence of the binder and diffusion flame would be expected to mitigate this effect. Extinguishment by pressure decay has been studied in the past in the context of controllable solid motors. ${ }^{15}$

\section{Conclusions}

A nonlinear combustion response model for AP has been developed as a building block for use in a composite propellant model to be coupled with a two-dimensional analysis of solid rocket motor flowfields. It is recommended that modelers first validate their model with steady-state data, validate the numerical method with classicallinear theory, and then progress to study of the mechanisms, properties, and features of their model.

The mechanistic role of condensed phase vis-à-vis gas-phase reactions as determinative of instability has been derived, with practical implications for desirable properties of advanced energetic ingredients. The peakedness of linear combustion response curves has been shown to depend on the $B$ parameter and now more specifically on the relative dependenciesof the condensed-phaseheat release and the heat feedback from the flame on burning rate. Stability favors a less exothermic solid phase that is more dependent on burn rate, implying that a lower activation energy is desired for the condensedphase reactions of energetic materials (so that they are less able to keep up with higher surface regression rates).

Analytic results also show the interrelationshipbetween intrinsic instability, deflagration limits, and point of exothermic heat release, with practical implications for energetic ingredients that exhibit extinction properties. The model predicts an increase in peak pressurecoupled response with increasing pressure, but can also be adjusted to explain an apparent increase in the peak response with decreasing pressure at low pressures if that is a real effect. The explanation is in terms of intrinsic instability limits, which can be approached at both very high and very low pressures as either gas-phase reactions become dominant (high pressure) or condensed-phase reactions become dominant (low pressure). Either extreme involves reactionsessentially at the surface, strongly coupling the heat release to the surface temperature. The Arrhenius decomposition kinetics produces a sensitive surface temperature that requires high accuracy in the numerical method. The low-pressure limit may also be an explanation for the low-pressure deflagration limit of AP at about $20 \mathrm{~atm}$.

Both model and data show peak response functions in excess of 4, reflecting the high-pressure exponent of AP together with its underlying combustion properties. More response function data at higher and lower pressures are recommended to better discern these properties.

The nonlinear code shows key features of amplitude and waveform effects. The nonlinear pressure-coupledresponse does not differ significantly from the linear response for small disturbances of practical interest, supporting the notion that crossflow disturbances are necessary to evoke combustion waveforms that are qualitatively different and for triggering nonlinear instabilities. Sufficiently large pressure oscillations, as from fully developed instabilities, will affect the response magnitudes but not the qualitative nature of the response unless so large as to initiate an extinguishment. It appears that AP can be extinguished more easily than a composite propellant. If the nonlinear response being larger than the linear response holds true for propellants, equilibration of amplitudes in motors will depend more on nonlinear energy losses.

\section{Acknowledgments}

This work is sponsored in part by the California Institute of Technology and in part by the Office of Naval Research Multidisciplinary University Research Initiative (MURI) under Grant N00014-95-I1338. MURI is a research program coordinating multiple scientific and engineering disciplines among many universities, in this case on the topic of combustion instability, managed by the Office of Naval Research. Judah Goldwasser is the Navy Program Manager.

\section{References}

${ }^{1}$ Baum, J. D., and Levine, J. N., "Modeling of Nonlinear Combustion Instability in Solid Propellant Rocket Motors," U.S. Air Force Research Lab., Rept. AFRPL-TR-83-058, Edwards AFB, CA, Feb. 1984. 
${ }^{2}$ Cohen, N. S., and Strand, L. D., "Improved Model for the Combustion of AP Composite Propellants," AIAA Journal, Vol. 20, No. 12, 1982, pp. 17391746.

${ }^{3}$ Cohen, N. S., and Strand, L. D., "Combustion Response to Compositional Fluctuations," AIAA Journal, Vol. 23, No. 5, 1985, pp. 760767.

${ }^{4}$ Cohen, N. S., and Strand, L. D., "Effect of AP Particle Size on Combustion Response to Crossflow," AIAA Journal, Vol. 23, No. 5, 1985, pp. 776780.

${ }^{5}$ Beddini, R. A., "Role of Turbulence Interactions in Solid Propellant Combustion Instability," AIAA Paper 98-3703, July 1998.

${ }^{6}$ Finlinson, J. C., Stalnaker, R. A., and Blomshield, F. S., "Ultra Pure Ammonium Perchlorate T-Burner Pressure Coupled Response at 500, 1000, and 1800 psi," AIAA Paper 98-3554, July 1998.

${ }^{7}$ Price, C. F., Boggs, T. L., and Derr, R. L., "The Steady-State Behavior of Ammonium Perchlorate and HMX," AIAA Paper 79-0164, Jan. 1979.

${ }^{8}$ Culick, F. E. C., "Review of Calculations for Unsteady Burning of a Solid Propellant," AIAA Journal, Vol. 6, No. 12, 1968, pp. 2241-2255.

${ }^{9}$ Beckstead, M. W., Derr, R. L., and Price, C. F., "Model of Solid Propellant Combustion Based on Multiple Flames," AIAA Journal, Vol. 8, No. 12, 1970, pp. 2200-2207.
${ }^{10}$ Beckstead, M. W., and Hightower, J. D., "Surface Temperature of Deflagrating Ammonium Perchlorate Crystals," AIAA Journal, Vol. 5, No. 10, 1967, pp. 1785-1790.

${ }^{11}$ Kooker, D. E., and Nelson, C. W., "Numerical Solution of Solid Propellant Transient Combustion," Journal of Heat Transfer, Vol. 101, No. 2, 1979, pp. 359-364.

${ }^{12}$ Levine, J. N., and Culick, F. E. C., "Nonlinear Analysis of Solid Rocket Combustion Instability,"Ultrasystems, Inc. (defunct), U.S. Air Force Research Lab., Rept. AFRPL-TR-74-45, Edwards AFB, CA, Oct. 1974.

${ }^{13}$ Culick, F. E. C., "Combustion Instabilities: Mating Dance of Kinetics, Combustion and Acoustics," AIAA Paper 2000-3178, July 2000.

${ }^{14}$ Culick, F. E. C., "Stability of Longitudinal Oscillations with Pressure and Velocity Coupling in a Solid Propellant Rocket," Combustion Science and Technology, Vol. 2, 1970, No. 4, pp. 179-201.

${ }^{15}$ DeLuca, L., "Extinction Theories and Experiments," Fundamentals of Solid-Propellant Combustion, edited by K. K. Kuo and M. Summerfield, Vol. 90, Progress in Astronautics and Aeronautics, AIAA, New York, 1984, pp. 661-732.

S. K. Aggarwal Associate Editor 